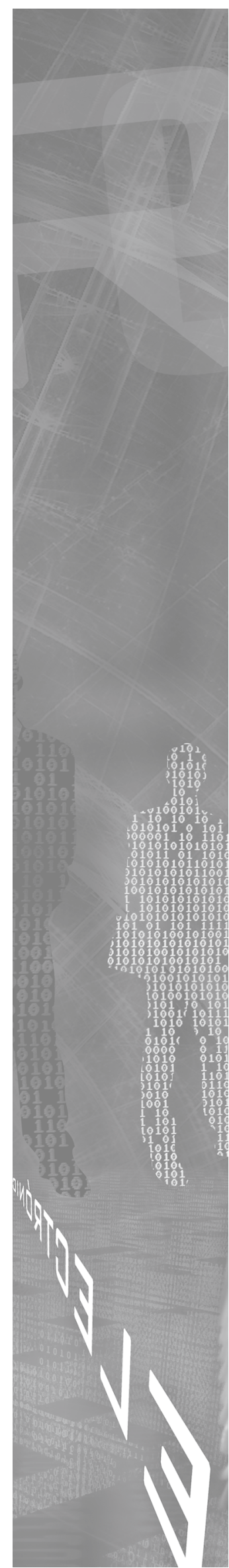

\title{
El razonamiento
}

espacial y la expresión gráfica bidimensional como experiencia a través de la papiroflexia

Luis Alberto Martínez C.

Recibido el 6 de marzo de 2009. Aprobado el 5 de mayo de 2009

\begin{abstract}
Resumen
Las nuevas exigencias que impone el entendido "desarrollo global", exigen la implementación de políticas educativas que propendan por la excelencia Académica, convirtiéndose esta última en elemento de especial controversia ya que se debe entender no como un concepto sino más bien como una referencia social e institucional. Con base en esta postura, las búsquedas pedagógicas planteadas desde la Decanatura y la Secretaría Académica de la Facultad de Ingeniería de la Corporación Universitaria Minuto de Dios (UNIMINUTO), especialmente a la indagación de nuevas formas de enseñanza y recursos pertinentes a los existentes desde lo conceptual, especificamente para este caso, con miras a la adquisición de habilidades matemáticas y de razonamiento espacial, condujeron a la puesta en practica de una "Prueba Piloto" con estudiantes de la Facultad de Ingeniería, Programa de Ingeniería Industrial, fundamentada en el uso de la Papiroflexia -Origami- como recurso didáctico para la enseñanza de la Geometría y el Dibujo bidimensional convirtiendo la experiencia en un trabajo de carácter teórico-práctico. De dicha experiencia desarrollada en el segundo semestre del año 2008, en el presente documento se hacen referencia los beneficios y cualidades, habilidades desarrolladas, contenidos trabajados así como los resultados obtenidos.
\end{abstract}

\section{Palabras clave}

Papiroflexia, Origami, Kirigami, Isometría, Normas ISO, AutoCAD.

\begin{abstract}
The new requirements that impose the understood "global development" demand the introduction of educative politics that tend by the academic excellence, furning it in an element of special controversy because it must not be understood as a concept, but as a social and institutional reference. On the basis of this position, the pedagogical searches planned by the Decanatura and the Academic Office of the Engineering Faculty of Universidad Minuto de Dios, specially to the adaptation of new ways of teaching and relevant resources to the ones that were specifically from the conceptual in this case looking to the acquisition of mathematic abilities and spatial reasoning let to the practice of a "Pilot Test" with students of the Engineering Faculty, Program of Industrial, based on the ace ok Papiroflexia-Origami as a didactic resource for the teaching of Geometry and bidimensional drawing turning the experience in a work of theoretic-practical character. About this experience developed in the second semester of 2008, the present document tells about the benefits and qualities, the abilities developed and the contents worked and the results obtained
\end{abstract}

\section{Key words}

Papiroflexia, Origami, Kirigami, Isometría, Normas ISO, AutoCAD. 


\section{Introducción}

La Papiroflexia u Origami, son las palabras con la cual se denomina la técnica consistente en la manipulación del papel a través de sus posibilidades de plegado para lograr determinadas figuras, las más conocidas, de índole zoomorfa. Esta condición ha conllevado a que tradicionalmente se le considere básicamente un entretenimiento o pasatiempo, sin embargo esta resulta una aproximación simple, dadas las múltiples consideraciones que se hallan implícitas.

En este sentido, resulta importante establecer que la Papiroflexia -Origami- es antes que nada una disciplina, considerada como arte-educativo a través del cual las personas pueden desarrollar su expresión artística, pero también es clara su relación directa con nociones espaciales y geométricas lo que ha conducido que en los últimos años se le haya prestado una renovada atención, procurando darle una mirada distinta, especialmente desde el punto de vista matemático y científico. Así, resulta interesante la forma en que 500 años después de haberse llevado a cabo el planteamiento de las leyes matemáticas de la perspectiva, actualmente se estudie el modo en que la Papiroflexia responde a una lógica dimensional precisa que a través de algoritmos describe el modo en que deben realizarse determinados pliegues a fin de lograr la figura que se desea y no menos que esto, la búsqueda de las ecuaciones matemáticas que explicitan lo que en la practica se hace sobre el papel.

\section{El concepto de la experiencia}

Tomando en consideración que la Expresión Gráfica en Ingeniería se ha fundamentado en la Geometría Descriptiva para el desarrollo de la habilidad espacial en el alumno y por tanto que este aprendizaje se halla sujeto a las limitaciones que la misma impone, especialmente a la doble labor que representa el pensar el espacio tridimensional operando sobre sus proyecciones bidimensionales en tanto que la capacidad de abstracción necesaria para este Razonamiento Espacial se convierte en condición básica y pilar fundamental a la hora de elaborar esquemas y planos, dicha habilidad representa uno de los objetivos más difíciles de conseguir ya que la misma se alcanza a lo largo de un proceso de aprendizaje que se inicia en la formación que se recibe desde la etapa infantil. De otra parte, si se toma como referencia el bajo rendimiento de los estudiantes de Colombia representado a través de los resultados de las pruebas internacionales TIMSS' se detectan errores conceptuales en Geometría, pero más relevante aun, se ha hecho evidente la poca habilidad a la hora de poner en práctica y en situaciones cotidianas la teoría recibida en la academia.

Por lo anterior, es que la búsqueda de alternativas pedagógicas que a través de la experiencia tangible se conviertan en recurso útil demostrando su aplicabilidad real, dan sentido y relevancia a la presente "Prueba". Con este propósito según Fogarty (1999)، para lograr altos niveles de aprendizaje es necesario "Armar una organización en la que se interactúe con el pensar" se consideró que el trabajo con las técnicas de la Papiroflexia -Origami- resultaba apropiada y consistente con las intenciones que la cátedra de dibujo planteaba y podría convertirse en un elemento de refuerzo y apoyo.

En este punto, imagínese una figura cualquiera desarrollada por medio de la técnica de la Papiroflexia, ¿Donde se hace evidente la Geometría? Pues bien, supóngase que para llegar a la figura en cuestión se parte de una hoja de papel con forma cuadrada, que a su vez se ha doblado por su diagonal, el cual, además es el eje de simetría y al mismo tiempo es hipotenusa de dos triángulos rectángulos iguales, y si finalmente, se doblan las esquinas se obtendrán más triángulos y al centro un hexágono; si se piensa en la situación anteriormente descrita se observará que no solamente se está plegando un papel sino que además se realiza un proceso mental organizado y sistemático que requiere de explicitar conceptos geométricos y matemáticos que van de lo simple a lo complejo y que además estimula la imaginación. Ejemplos de este tipo son los que se consideran útiles y pertinentes como herramienta didáctica y sobre los cuales se fundamenta el ejercicio aquí planteado.

\section{Descripción de la experiencia}

Durante el periodo lectivo correspondiente al segundo semestre de 2008, con los alumnos que conformaban 3 grupos de estudio, inscritos en la cátedra de Dibujo del programa de Ingeniería Industrial, correspondiente al nivel de Segundo Semestre se dio inicio a lo que se denominó "Prueba Piloto" para la implementación de alternativas pedagógicas en el caso del dibujo en Ingeniería a través de la Papiroflexia -Origami-. Se propusieron tres sesiones cuyo producto debería ir evolucionando en conceptualización y dificultad de ejecución con el fin además de hacerlo consecuente con las temáticas propias de la cátedra. Los productos que en este caso particular serian figuras tridimensionales de papel se escogieron y orientaron sobre la premisa que el Origami que

\footnotetext{
'TIMSS: "Estudio Internacional de Tendencias en Matemáticas y Clencias", Colombia participó en la evaluación correspondiente al año 2007 con una muestra de 9674 estudiantes.
} 
plantea mayor contenido desde la perspectiva de la geometría, es el de los "Modelos desarrollados a partir de módulos". Así, los modelos propuestos buscaron dar solución a figuras básicas que implicaran la comprensión tridimensional del objeto y la forma por medio de la manipulación-manufactura real y su posterior descripción a través del Dibujo Lineal y el Dibujo Asistido por Computador.

\section{Desarrollo de la experiencia}

Considerando que la experiencia propuesta incluía diversos contenidos, se estimó pertinente que su desarrollo se dividiera en dos fases claramente diferenciadas; de una parte se llevó a cabo un trabajo presencial y tutorado, cuyo objeto consistió, a través de las técnicas de la Papiroflexia -Origami- en la ejecución de cada uno de los poliedros seleccionados. Esta etapa implicó una aproximación teórica a las nociones más elementales de la Papiroflexia -Origami- como son los tipos de plegado y su simbología en los Algoritmos Gráficos, además del seguimiento del proceso por parte del profesor Francisco Alberto D'Achiardi experto en Papiroflexia.

El resultado de este trabajo se vió reflejado en la elaboración física de los distintos modelos y resultó en el punto de partida para la segunda fase de la "prueba", guiada por el Docente Luis Alberto Martínez Camacho la cual implicaba una continuación del trabajo, y donde el alumno ponía en práctica las nociones de representación gráfica bidi y tridimensional. La primera labor se hizo inmediatamente concluido el modelo de Papiroflexia -Origami- mediante el dibujo rápido de bocetos a mano alzada sin escala, en procura de la comprensión ágil de lo que se visualiza a modo de una primera aproximación que permita confrontar la realidad del objeto creado con la forma en que se está entendiendo y expresando. Sobre la discusión de los resultados se procede a un paso intermedio que implica la elaboración del modelo tridimensional, ejecutado esta vez sobre los criterios del Dibujo Geométrico y Lineal, dando uso a los instrumentos propios y el empleo de las técnicas necesarias, modos de representación otorgando una Escala y su descomposición en vistas principales norma ISO-A. Finalmente, el proceso llega a su conclusión mediante el modelado del objeto propuesto en programa AutoCAD. Esta integración de temáticas busca una integralidad del proceso de aprendizaje, a partir de una secuencia racional de contenidos desde lo conceptual-teórico a lo real-practico sobre la base de una Lógica Deductiva.

\section{Las sesiones}

Tal y como se ha venido insistiendo, el desarrollo de la denominada "Prueba Piloto" se realizó a partir de tres sesiones diferenciadas, cada una con distintos ejercicios y modos de evaluación. A continuación se incluyen de modo específico los cuadros Guía de Clase, con objetivos y desarrollo de cada una de las sesiones en mención.

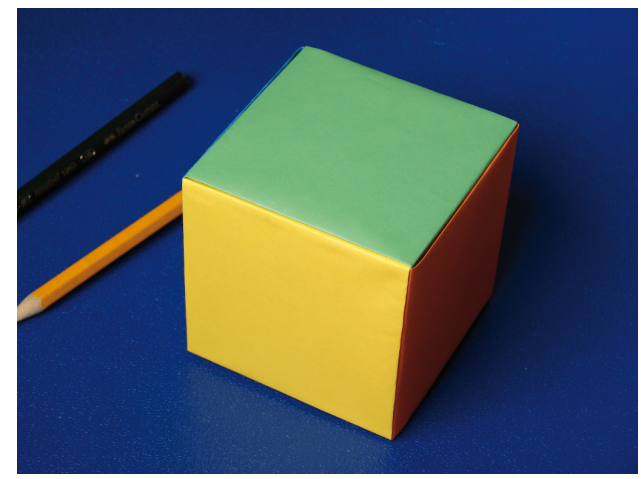

Figura 1. Cubo Hexa-Modular en Origami (Sesión 1), elaborado en clase. Fuente: El Autor, 2008.

SESIÓN No 1. -CUBO HEXA-MODULAR-

\begin{tabular}{|c|c|}
\hline Tema: & $\begin{array}{l}\text { Proyección Isométrica de un Cubo como resul- } \\
\text { tado de la solución con Papiroflexia -Origami- } \\
\text { del cubo Hexa-Modular. (Gráficos } 4 \text { y 5). }\end{array}$ \\
\hline Objetivos: & $\begin{array}{l}\text {-Plegar el cubo de David Mitchell (Papiroflexia- } \\
\text { Origami) realizando y ensamblando los mó- } \\
\text { dulos mediante el seguimiento del Algoritmo } \\
\text { Gráfico y las aclaraciones presénciales. } \\
\text { - Dibujar a mano alzada el Cubo resultante en } \\
\text { la práctica de Papiroflexia a modo de modelo } \\
\text { tridimensional, usando una visión monocular } \\
\text { estática. } \\
\text {-Proceder a resolver con instrumentos de dibu- } \\
\text { jo (Regla"T"-Juego de Escuadras) y a escala, el } \\
\text { dibujo realizado a mano alzada resultante en } \\
\text { Proyección Isométrica }\left(30^{\circ}\right) \text {. }\end{array}$ \\
\hline $\begin{array}{l}\text { Fecha de } \\
\text { Realización: }\end{array}$ & 16.09.2008. \\
\hline Duración: & Dos (2) Horas Clase. \\
\hline Contenidos: & $\begin{array}{l}\text {-Interpretación de Algoritmos Gráficos } \\
\text { (diagrama del módulo) y ensamblaje para el } \\
\text { "Cubo Mitchell". } \\
\text { - Convenciones para el desarrollo de plie- } \\
\text { gues y movimientos. } \\
\text { • Diferencia práctica entre visión estereoscó- } \\
\text { pica y visión monocular para desarrollar la } \\
\text { técnica de dibujo geométrico con modelo. } \\
\text { - Planos y líneas paralelas. }\end{array}$ \\
\hline Actividades: & $\begin{array}{l}\text { - Plegado del cubo Hexa-modular (seis módu- } \\
\text { los) de David Mitchell. } \\
\text { - Dibujo a mano alzada del modelo. } \\
\text { - Dibujo con instrumentos de una proyección } \\
\text { isométrica del cubo tomando como base el } \\
\text { dibujo a mano alzada. }\end{array}$ \\
\hline
\end{tabular}




\begin{tabular}{|l||l|}
\hline Evaluación: & $\begin{array}{l}\text { •Hacer el dibujo a mano alzada del modelo. } \\
\text { - Realización de un modelo isométrico a esca- } \\
\text { la 1:1 del cubo "Mitchell" resuelto. }\end{array}$ \\
\hline \hline Recursos: & $\begin{array}{l}\text {-Seis hojas de papel iris partido en cuadrados } \\
\text { de 15x15cm, de color distinto cada uno. } \\
\text { •Algoritmo Gráfico (diagrama) preparado para } \\
\text { la solución del cubo "Mitchell" } \\
\text { •Colbón. }\end{array}$ \\
\hline
\end{tabular}

Tabla 1. Guía de Clase, Sesión 1, Fuente: D’Achiardi - Martínez, 2008.

\section{SESIÓN No 2. -CUBO TRI-MODULAR-}

\begin{tabular}{|c|c|}
\hline Tema & $\begin{array}{l}\text { Vistas Principales y Auxiliares, Norma ISO-A a partir } \\
\text { de la construcción de parejas de planos parale- } \\
\text { los con el Cubo Tri-modular en Kirigami. }\end{array}$ \\
\hline Objetivos & $\begin{array}{l}\text {-Plegar el cubo Modular en Kirigami realizan- } \\
\text { do y ensamblando los módulos mediante el } \\
\text { seguimiento del algoritmo gráfico y aclaraciones } \\
\text { presénciales. } \\
\text { - Identificar en el modelo tridimensional cons- } \\
\text { truido por el alumno las tres parejas de planos } \\
\text { paralelos asignándole un color distintivo a cada } \\
\text { pareja. } \\
\text {-Dibujar con instrumentos la proyección isomé- } \\
\text { trica del cubo tri-modular y su descomposición } \\
\text { en las tres vistas principales (Horizontal, Vertical y } \\
\text { Lateral Izquierda). } \\
\text {-Modelar en AutoCAD, bajo los principios del } \\
\text { dibujo isométrico el cubo tri-modular y sus res- } \\
\text { pectivas vistas principales }\end{array}$ \\
\hline $\begin{array}{l}\text { Fecha de Reali- } \\
\text { zación: }\end{array}$ & 07.10 .2008 \\
\hline Duración: & Dos (2) Horas clase \\
\hline Contenidos: & $\begin{array}{l}\text { - Planos-Líneas paralelas y perpendiculares. } \\
\text { - Representación bidimensional de un objeto } \\
\text { tridimensional a partir de sus vistas principales } \\
\text { (Norma ISO-A). } \\
\text { - Dibujo Isométrico }\left(30^{\circ}\right) \text {. }\end{array}$ \\
\hline Actividades: & $\begin{array}{l}\text {-Plegado del cubo tri-modular en Kirigami. } \\
\text { - Identificación de parejas de planos paralelos } \\
\text { con aplicación de color sobre el plano } \\
\text { - Dibujo a mano alzada del modelo. } \\
\text { - Dibujo con instrumentos de la proyección } \\
\text { isométrica del cubo tri-modular acompañado } \\
\text { de su descomposición bidimensional en vistas } \\
\text { principales. } \\
\text {-Dibujo del modelo en AutoCAD. }\end{array}$ \\
\hline Evaluación: & $\begin{array}{l}\text {-Desarrollo del cubo tri-modular } \\
\text { - Representación tridimensional del cubo tri-mo- } \\
\text { dular a mano alzada y en clase. } \\
\text { - Dibujo del cubo tri-modular con instrumentos } \\
\text { (lápices HB y } 2 \mathrm{H} \text { ) en representación isométrica } \\
\text { (30) y a escala } 1: 1 \text { y su descomposición en } \\
\text { vistas principales de acuerdo a la Norma ISO-A. } \\
\text { - Modelado del Cubo Tri-modular en AutoCAD }\end{array}$ \\
\hline Recursos: & $\begin{array}{l}\text { - Dos hojas de papel cartulina partido en cua- } \\
\text { drados de } 20 \text { X } 20 \mathrm{~cm} \text {. } \\
\text { - Algoritmo gráfico (diagrama) para el desarrollo } \\
\text { del Cubo tri-modular. } \\
\text { - Tijeras } \\
\text { - Colbón. } \\
\text { - Plumígrafos o marcadores en tres colores } \\
\text { diferentes } \\
\text { - Regla "T" } 50 \mathrm{cms} \text {, Juego de escuadras ( } 30-60^{\circ} \\
\text { y } 45^{\circ} \text { ) }\end{array}$ \\
\hline
\end{tabular}

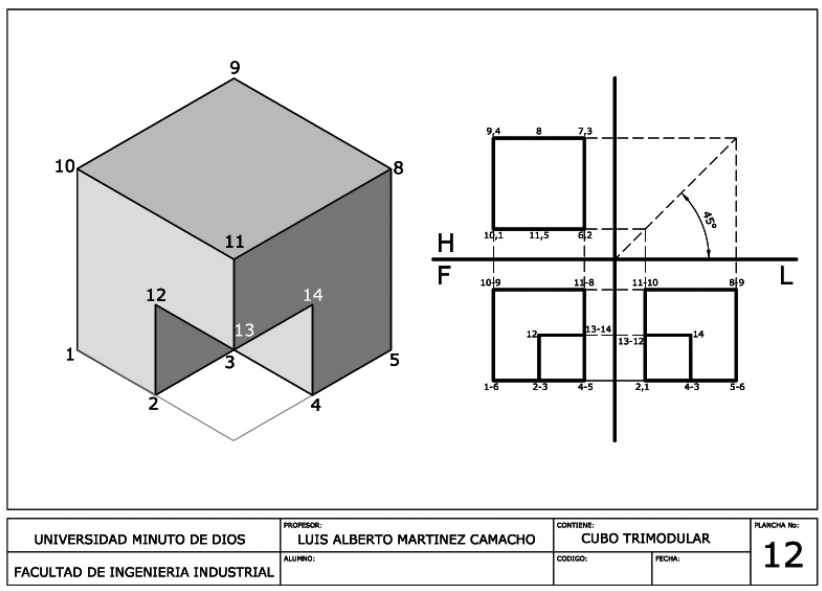

Figura 2. Cubo Tri-modular, Isométrico y Vistas principales. Fuente: El Autor, 2008.

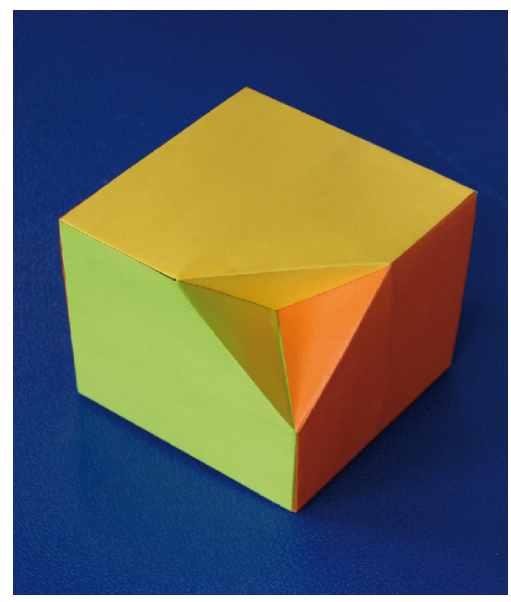

Figura 3. Cubo Columbus en Origami (Sesión 3), elaborado en clase. Fuente: El Autor, 2008.

\section{SESIÓN No 3. -CUBO COLUMBUS.}

\begin{tabular}{|c|c|}
\hline Tema: & $\begin{array}{l}\text { Dibujo isométrico de figura con extrución en una de } \\
\text { sus aristas a partir del desarrollo del Cubo Colum- } \\
\text { bus (David Mitchell) y la pirámide Extruida (Francisco } \\
\text { D'Áchiardi). (Gráficos } 8 \text { y 9). Pre-requisito: Haber reali- } \\
\text { zado el Cubo Hexa-modular (Sesión 1) de David Mi- } \\
\text { tchell. }\end{array}$ \\
\hline Objetivos: & $\begin{array}{l}\text { - Plegar el cubo Columbus realizando y ensamblan- } \\
\text { do los módulos mediante el seguimiento del algorit- } \\
\text { mo gráfico y aclaraciones presénciales. } \\
\text { - Realizar en Papiroflexia -Origami- el instrumento para } \\
\text { medir un ángulo de } 60^{\circ} \\
\text { - Plegar con dos módulos especulares la pirámide de } \\
\text { base triangular y lados isósceles de } 90^{\circ} \text { para ser extrui- } \\
\text { da del Cubo Columbus. } \\
\text { - Trazado con instrumentos de la proyección isométri- } \\
\text { ca del cubo con el espacio de la extrución libre. } \\
\text { - Modelado del resultado isométrico en programa Au- } \\
\text { toCAD, definiendo en el "Espacio Papel" rotulo y darle } \\
\text { escala } 1: 1 \text {. }\end{array}$ \\
\hline $\begin{array}{l}\text { Fecha de Reali- } \\
\text { zación: }\end{array}$ & 18.11 .2008 \\
\hline Duración: & Dos (2) Horas clase \\
\hline
\end{tabular}




\begin{tabular}{|c|c|}
\hline Contenidos: & 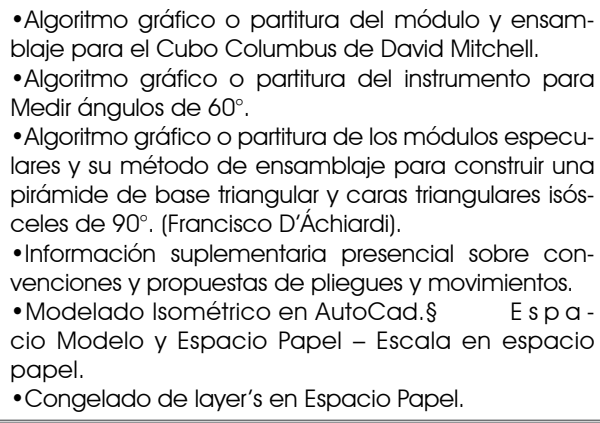 \\
\hline Actividades: & $\begin{array}{l}\text { - Plegado de un instrumento en papel para medir } \\
\text { ángulos de } 60^{\circ} \\
\text { - Plegado del cubo Columbus } \\
\text { - Identificación del espacio o vacío en el cubo. } \\
\text { - Dibujo con instrumentos de una proyección } \\
\text { isométrica del cubo columbus con su extrución } \\
\text { piramidal para comparar con la mano alzada. } \\
\text { - Modelado AutoCAD en Espacio Papel del modelo } \\
\text { Cubo Columbus y edición en Espacio Papel a Esca- } \\
\text { la } 1: 1 \text {, rotulo del curso tamaño DIN A-4 e impresión } \\
\text { final. }\end{array}$ \\
\hline Evaluación: & $\begin{array}{l}\text { Entrega del modelo realizado en AutoCAD (Espacio } \\
\text { Papel) formato digital e Impresión en Papel formato } \\
\text { DIN A-4, acompañado del modelo de Papiroflexia - } \\
\text { Origami-. }\end{array}$ \\
\hline
\end{tabular}

Tabla 3. Guía de Clase, Sesión 3, Fuente: D’Achiardi - Martínez, 2008.

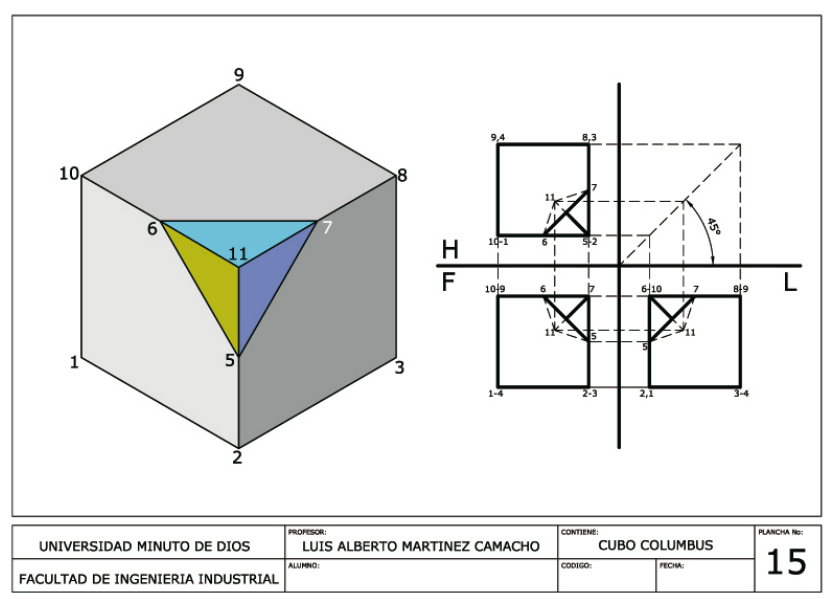

Figura 4. Cubo Columbus, Isométrico y Vistas principales. Fuente: El Autor, 2008.

\section{Vl. Evaluación de resultados}

\section{Ventajas:}

Generar modelos tridimensionales que pueden ser sometidos a análisis en la realidad; estos a su vez facilitan la representación gráfica, especialmente si se tiene en cuenta que el mismo modelo requiere de una conceptualización previa para su ejecución, la misma que potencia la habilidad de comprensión espacial en términos generales. Además no se puede desconocer que la Papiroflexia -Origami- es una alternativa óptima (barata) desde la óptica económica para la producción de modelos tridimensionales que generalmente resultan costosos.

Por tratarse de un ejercicio que se ejecuta en secuencia lineal (con base en algoritmos gráficos) es notable que los alumnos resuelvan la problemática presentada con altos niveles de certeza ya que su solución se conoce de antemano y no genera dudas del resultado que se debe alcanzar; en este sentido se describe un proceso que se podría definir como una "selección" de recursos de acuerdo con su propia comprensión, el cual, permite a cada uno la materialización del objeto. En otras palabras, se puede decir que el alumno encuentra su propio proceso particular a partir de la propuesta en un seguimiento secuencial de pasos, con la característica singular que radica en el hecho que el producto tendrá existencia real y por tanto se convierte en un modelo analizable y evaluable por parte del mismo estudiante. Se trata entonces de un proceso que produce resultados inmediatos, dada la elaboración del modelo, pero se hacen permanentes en la medida que el mismo se utilizará en la continuación del análisis y desarrollo del proceso. Esta instancia resulta opuesta a los métodos tradicionales de enseñanza del razonamiento espacial ya que estos se basan sobre esquemas que necesariamente requieren de altos niveles de abstracción que acá son reemplazados por los modelos creados.

Resultó notable también, la manifestación de "solidaridad" para la efectiva y correcta solución de los ejercicios entre compañeros. Sobre este aspecto, se puede afirmar que al tratarse de un producto que es real y visible, de parte de cada uno de los aprendices se manifiesta un afán por la conclusión del objetivo y en los casos en que se presentan dificultades de comprensión o de ejecución hay una postura más abierta para solicitar colaboración de aquellos que resultan "adelantados" en el proceso. Lo anterior implica entre otras cosas que el conocimiento se puede convertir en un esfuerzo colectivo, instancia que puede resultar de especial interés en la medida que el trabajo en equipo es una de las habilidades que deberían estimularse, como es bien sabido, no solamente a niveles académicos sino también de la sociedad en general.

El hecho que la mecánica pedagógica en este caso se perciba ante todo como un juego, también se convierte en una actitud mas desinhibida frente al conocimiento que tiene como consecuencia inmediata la exploración del alumno respecto de las posibilidades del producto alcanzado. En este sentido vale la pena destacar de manera especial los resul- 
tados del ejercicio final (Sesión 3, Cubo Columbus). En este caso se recurre a la solución de una figura "cubo" que presenta una extrusión de una de sus esquinas que siendo resultado de una serie de encajes autosostenidos se convierte a su vez en un módulo cúbico que posibilita el ensamblaje de varios de estos cubos Columbus en figuras más complejas. En la medida en que cada uno iba dando por concluido su objeto individual de manera espontánea, proponía asociarlo con los demás que iban apareciendo encontrando soluciones de enorme interés, ya que en opinión de los docentes acompañantes del proceso abren la posibilidad de una aproximación distinta en la comprensión de temáticas complejas como puede ser la "Teoría del Caos" y por ende de la "Geometría Fractal" ya que por sus caracteristicas y teóricamente, estos elementos modulares podrían ser reproducidos de manera infinita.

\section{Desventajas:}

Pueden llegar a existir diferencias importantes en el desarrollo de los ejercicios y por consecuencia de los resultados que se obtengan con los modelos tridimensionales en razón de las disparidades referentes a las habilidades manuales con que cada alumno cuente, al mismo modo que con la disciplina con la que se asume ya que es innegable que se requiere niveles de atención y especialmente disposición a seguir instrucciones especificas en pro de la eficiencia en términos de tiempo.

Es importante relacionar de modo directo los contenidos de la Cátedra que se imparte con la propuesta de la Papiroflexia -Origami- porque se corre el riesgo de no lograr un vínculo claro y eficiente entre los contenidos. En este sentido, la escogencia de los modelos a ejecutar es vital ya que, de una parte, si el ejercicio resulta demasiado complejo podría resultar contraproducente en la medida en que se convierte en un reto la solución, pero si resulta demasiado sencillo no resulta clarificador de conceptos.

Se comprobó que el trabajo con grupos grandes resulta menos beneficioso; sobre esta cuestión es importante anotar que de los tres grupos estudio, dos eran conformados en promedio por 30 estudiantes, mientras que el tercero contaba con 11 alumnos. Lo que se pudo observar en este sentido es que los niveles de atención y por tanto de eficiencia-rapidez en la solución de los modelos se veían determinados por dicha variable.

\section{Sugerencias:}

Resulta importante implementar de modo consistente y secuencial experiencias de este tipo que permitan continuar el proceso de indagación sobre las posibilidades que pueda representar el empleo de técnicas de Papiroflexia -Origami- para la adquisición o perfeccionamiento de habilidades profesionales. La "Prueba Piloto", llevada a cabo permitió comprobar la relación entre el razonamiento espacial y abstracto y los medios que se empleen para facilitarlo, sin embargo se vislumbran otras posibilidades que se presentan en un espectro tan amplio que va desde las demostraciones matemáticas que se hallan implícitas en las figuras, comprensión y cálculo del volumen de sólidos en revolución, explicación y comprensión de la Geometría Fractal y Teoría del Caos, (mismas que hallan relevancia para disciplinas relacionadas con la Informática y los Sistemas) hasta estudios sociales del impacto que sobre los colectivos humanos puede tener el uso e implementación de las técnicas en mención.

Operativamente, es claro que resulta en mejorías importantes la aplicación de la Papiroflexia -Origami- en grupos que no sean numerosos. Tal y como se comentó con anterioridad se pudo detectar que la atención y disciplina se ven considerablemente incrementados en grupos de tamaño medio, considerándose los grupos de 15 participantes los ideales para el desarrollo óptimo de la experiencia.

\section{VII. conclusiones}

- El empleo de las técnicas de la Papiroflexia permite establecer una relación beneficiosa entre habilidades, destrezas y el conocimiento con el cual los alumnos cuentan en los niveles de inicio de carrera, convirtiéndose en un ejercicio reflexivo ya que si bien de una parte se generan habilidades manuales, de otra se logra por medio de un modo gráfico y ameno el desarrollo de la necesaria concepción volumétrica sin desconocer además el hecho de fomentar la agilidad mental y el desarrollo de estrategias que permitan la solución de problemas lógicos y/o matemáticos donde la clave para utilizar pedagógicamente la papiroflexia está en interpretar geométricamente y de modo simultaneo qué hacemos cuando doblamos papel.

- El estudio de los objetos "concretos" necesariamente implica un nivel de observación que facilita considerablemente el proceso de abstracción de los mismos, además que se convierte en una propuesta innovadora para la producción de conocimiento e importante apoyo al momento de la correcta comprensión de conceptos, relaciones y métodos ya que posibilita una enseñanza activa.

- De modo consistente con la propuesta desarrollada, la formación geométrica, de comprensión es- 
pacial y representación gráfica que se logra es valiosa en la medida que proporciona un desarrollo y evolución desde la percepción visual a la espacial convirtiéndose en un medio que posibilita ejercitar y mejorar habilidades generales del pensamiento donde el ejercicio de visualización, comparación y representación se convierte en una aproximación integral a la geometría y la matemática.

\section{Referencias}

[1] Bascetta, P. (1998). Origami: Geometría con la carta (I). Revista Quadrato mágico, (52). Recuperado el 5 de Abril de 2009, de http://www.origami-cdo. it/articoli/artgeo.htm

[2] Fiell, Ch. \& P. (2003). El diseño del Siglo XX. Madrid, España: Ed. Tashen.

[3] Fogarty, R. (1999). "Architects of the Intellect", Educational Leadership, Vol. 57, No 3.

[4] Fusé, T. (2000). Unit origami: Multidimensional transformations. Tokyo: Japan Publications.
[5] Gonzáles, J. (1991). Como hacer figuras de papel, Iniciación a la Papiroflexia. Madrid, España: Ediciones Hermann Blume.

[6] Jackson, P. (1998). Enciclopedia del origami y artesanía de papel. Barcelona, España: Ed. Acanto.

[7] Koestler, A. (1998). En busca de lo absoluto. Barcelona, España: Ed. Kairos.

[8] Macchi, P. \& Scaburri, P. (1997). Nuevos objetos de papiroflexia. Barcelona, España: Editorial De Vecchi.

[9] Página oficial de la Asociación Española de Papiroflexia. Recuperado el 10 de Febrero de 2009, de http://www.pajarita.org

[10] Royo, J. I., Recuperado el 4 de Marzo de 2009, de http://xtsunxet.usc.es/royoprieto

[1 1] Palacios, V. (1986). La creación en papiroflexia. Barcelona, España: Ed. Salvatella.

[12] Root-Bernstein, R. y M. (2000). El secreto de la creatividad. Barcelona, España: Ed. Kairos.

[13] Westheimer, M. (1991). El pensamiento productivo. Cognición y desarrollo humano. Barcelona, España: Ed. Paidos.

Luis Alberto Martínez C. Arquitecto, Universidad Piloto de Colombia, 2000, Candidato al título de "Magíster en Planificación Urbana y Regional", Pontificia Universidad Javeriana 2009, Bogotá. En la actualidad, es docente de la Facultad de Ingeniería de la Corporación Universitaria Minuto de Dios (UNIMINUTO).lamartinez@uniminuto.edu 